\title{
Nonlinear Wave Phenomena in Coasting Beams
}

\author{
P. L. Colestock, L. K. Spentzouris and F. Ostiguy \\ Fermi National Accelerator Laboratory ${ }^{\dagger}$ \\ P. O. Box 500, Batavia, IL 60510, USA
}

\begin{abstract}
In beams which are suf®ciently close to the linear stability limit, a variety of nonlinear wave phenomena are readily observed which can be used to diagnose aspects of the beam dynamics and the machine impedance. We have found that debunched beams in both the Fermilab Main Ring and the Tevatron are marginally stable to longitudinal oscillations and exhibit nonlinear three-wave coupling as well as nonlinear Landau damping and the formation of soliton-like perturbations. In addition, we have generated classical nonlinear echoes using twofrequency excitation in the Fermilab Accumulator. These phenomena can be used as diagnostic tools to determine diffusion rates in the beam as well as the longitudinal impedance. Moreover, aspects of these effects are likely involved in the approach to equilibrium as a longitudinal instability saturates under the in-uence of a driving impedance. We present experimental results and analytical models based on perturbation techniques. In addition, we describe particle simulations which model the fully nonlinear evolution of these phenomena.
\end{abstract}

\section{Introduction}

In recent years, growing attention has been given to the issue of increasing beam intensity in Fermilab's synchrotrons. However, as these machines have been pushed to operate near their stability limits, they have been observed to be operating under marginally stable conditions.[1] A variety of unstable modes have been identi®ed and suppressed to some degree, however there exists a background level of 'uctuations under nominally quiescent conditions whose origins have not been well understood.

To shed further light on these phenomena, an investigation has been underway to determine the driving causes and saturation mechanisms associated with potential instabilities in Fermilab machines using a variety of methods including the wellknown beam transfer function technique [2]. In this procedure, an externally-applied sinusoidal voltage is impressed on the beam and the response is monitored with a wall current pickup elsewhere in the ring. The magnitude and phase of the response

${ }^{\dagger}$ Operated by the Universities Research Association,Inc, under contract with the U.S. Department of Energy is a measure of the beam distribution function and the machine impedance at the excited frequency. For ease of analysis, our efforts have been focussed on the study of longitudinal oscillations in an initially fully debunched beam.

However, under certain conditions, beam transfer function measurements have exhibited nonlinear behavior, indicating a rich variety of wave phenomena not normally considered in the beam transfer function model. In fact, the ease with which these phenomena can be made to occur suggests a ubiquitous presence in any beams which are driven near their stability limits. Moreover, in the course of this study, we have rediscovered a weakly nonlinear coupling phenomenon which has been investigated in other ßelds, but has not yet been exploited in high energy synchrotrons: beam echoes. Echo phenomena carry the potential as a very useful diagnostic of weak diffusion phenomena in the beam.

In this work we ${ }^{\circledR}$ rst describe the beam transfer function technique in the context of the Fermilab machines in which these measurements were made. Then we outline a series of experiments carried out to clarify the nature of the observed modemode coupling. Next, we describe the observations of longitudinal beam echoes and give an explanation of these phenomena in terms of a weakly- nonlinear mode coupling model. Finally, we report the results of particle simulations which were undertaken to describe the fully nonlinear evolution of the mode coupling. In particular, we wish to shed light on how the beam emittance is affected by the presence of coherent -uctuations and the manner in which an equilibrium state is approached.

\section{Experimental Results}

In both the Fermilab Main Ring and Tevatron, attempts at measuring beam transfer functions have led to rather complex results[1]. Regarding measurements in the Main Ring, a $5 \mathrm{MHz}$ resonant cavity with a $\mathrm{Q}$ of 42 was used as a kicker. In this case, the beam transfer function showed the presence of notches in the response function which presumably correspond to depletion zones in the momentum distribution. In the response, each notch represents a narrow-band resonance which adds a degree of freedom to the longitudinal mode structure. Further investigation showed that the momentum location of the notches was tied to the betatron tune settings in the machine, which implies 


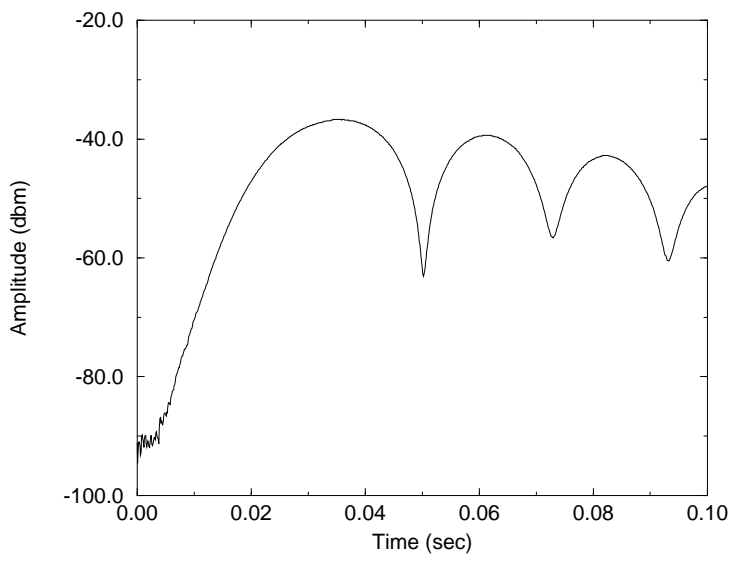

Figure 1: Oscillation of the drive amplitude following a 0.5 msec burst of rf at the $h=106$ harmonic. The current envelope suggests phase rotation.

the notches are the result of transverse resonances which deplete momentum space depending on the level of machine chromaticity. This notion was con®rmed by changing tune settings, resulting in a systematic movement of the momentum notches. As a result of these notches, it is expected that the linear stability boundary for longitudinal oscillations is radically altered. In particular, a typical momentum width of a notch was found to be 5\% or less of the full momentum spread. The Keil-Schnell stability criterion [3] suggests that such a notch would reduce the stability threshold by over two orders of magnitude.

In an attempt to assess stability in this case, the full spectral response over several revolution harmonics was measured. However, it was found that the response in the Main Ring was signi®cantly nonlinear with no apparent intensity threshold. (Similar results have been previously reported for high intensity coasting beams in the Tevatron albeit with a well-de®nd threshold condition[4]). A key feature in the response is the observed tendency of the drive frequency to couple primarily downward in frequency. In fact, the coupling to the sidebands was observed to produce a regular cascade to increasingly lower frequencies. In order to further clarify these phenomena, a series of experiments in the time domain was carried out where the kicker cavity was excited for a short period of time, approximately $0.5 \mathrm{msec}$, using a fast rf gate. It is worthwhile to note that the $5 \mathrm{MHz}$ cavity ${ }^{\circledR}$ elds decay in $0.01 \mathrm{msec}$. The response at the ${ }^{\circledR}$ rst lower sideband, shown in Fig. 1 shows a characteristic oscillation of the beam current envelope following the excitation pulse suggestive of phase rotation. In addition, lower sidebands showed the same response after a delay proportional to the harmonic spacing relative to the drive frequency. The observed time delay suggests a clear causal relationship in the ${ }^{-}$ow of power from higher harmonics to lower ones and implies that the coupling occurs predominantly across one harmonic at a time. Of note here is the fact that the peak response occurs several harmonics distant from the drive fre- quency. This characteristic is intrinsic to the beam response and is not a function of the applied power.

\section{Parametric Coupling Model}

These phenomena are characteristic of classical three-wave, or parametric, coupling which has been studied extensively in plasma physics[5]. Both the single-sided character of the frequency response and the depletion of the pump frequency are well-known effects in this type of resonant coupling. Unique to the beam case is the fact that there exists an in ${ }^{\circledR}$ nite collection of longitudinal modes, and hence potential daughter waves, in this periodic system.

The conditions for longitudinal mode coupling can be obtained from the Vlasov equation by a perturbation approach wherein mode-mixing terms are retained in the expansion of the distribution function. Conservation of energy leads to the frequency matching condition, $\omega_{n}+\omega_{k}=\omega_{m}$. If the frequency of the driving wave is $\Omega_{0}$, then $\omega_{n}+\Omega_{0}=\omega_{m}$ must be obeyed in order to have energy transferred into a pair of longitudinal modes. This condition ensures that coupling occurs only downward in frequency. The dispersion relation for parametric coupling of three waves can be shown to be[4]:

$$
\begin{gathered}
\nu^{2} \int \frac{d \epsilon \frac{\partial f_{0}}{\partial \epsilon}}{\left(\omega_{m}-m \gamma\right)^{2}} \int \frac{d \epsilon \frac{\partial f_{0}}{\partial \epsilon}}{\left(\omega_{n}-n \gamma\right)^{2}}= \\
\left(\frac{e \omega_{0}}{2 \pi}\right) \cdot \int \frac{d \epsilon \frac{\partial f_{0}}{\partial \epsilon}}{\left(\omega_{n}-n \gamma\right)\left(\omega_{m}-m \gamma\right)^{2}} \times \\
\int \frac{d \epsilon \frac{\partial f_{0}}{\partial \epsilon}}{\left(\omega_{m}-m \gamma\right)\left(\omega_{n}-n \gamma\right)^{2}}
\end{gathered}
$$

where $\nu$ is the growth rate and $\gamma=k_{o} \epsilon$. We note that the left hand side of Eq. 1 represents the product of linear dispersion relations for modes $m$ and $n$. Thus, the effect of a small driving amplitude is that the coupling threshold must occur close to the linear mode frequencies. Moreover, the threshold for coupling decreases to zero amplitude as the linear stability boundary is approached. The coupling threshold is found to scale as the inverse fourth power of the momentum spread so that coupling is most likely to occur in notches in the distribution function.

In this analytical model, the pump amplitude was held ${ }^{\circledR x e d}$, however it can be expected that the daughter waves will also have a reaction back on the pump. In simple systems, it has been shown that the exchange of energy between parametrically coupled waves reaches a steady state where all the energy oscillates between the various modes [6]. Indeed the pump may become completely depleted by the presence of stronglycoupled daughter waves. Such a process is likely the cause of the enhanced wave amplitudes at lower sidebands relative to the pump amplitude. With few exceptions, however, the case of a large number of multiple harmonics, including dissipation, has not been treated in the literature [6]. 


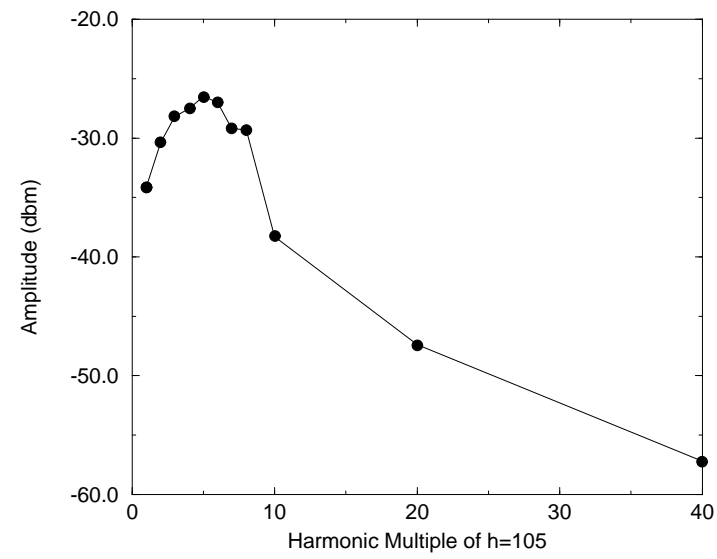

Figure 2: Harmonic spectrum generated in the beam response to impulse excitation at $\mathrm{h}=106$. The harmonics shown here are associated with the ${ }^{\circ}$ rst lower sideband, $h=105$, excited in the parametric decay.

\section{Nonlinear Landau Damping}

The oscillatory behavior of the impulse response observed in the Main Ring can be related to a well-known phenomenon in plasma physics known as nonlinear Landau damping[7]. In this process, an initial sinusoidal potential perturbation will cause a portion of the distribution function to become trapped in the potential wells of the wave, namely those particles whose relative energy in the frame moving with the perturbation is suf®ciently small. This, of course, causes bunching to occur and the resulting trapped particles will begin to exchange energy coherently with the wave in the form of a rotation in phase space. The steady state condition reached is such that the oscillations of the system decay much more slowly that that expected from simple decoherence through the frequency dispersion of the beam. This is indeed the case, as shown in Fig. 1. Another characteristic of this process is the tendency to generate a rich higher harmonic spectrum as the trapped particles are progressively focussed into tighter and tighter bunches[8]. This feature was also observed, as depicted in Fig. 2. This suggests that wake®elds play a role in the evolution of the transient response and we wish to exploit this connection to determine the wake $囚$ elds themselves.

\section{Beam Echoes}

One application which arises from the phenomenon of parametric coupling in the time domain is that of beam echoes[9]. Echoes have been found in many other ®elds[10, 11], but have not yet been applied in the context of synchrotrons, although bunched beam echoes have been investigated theoretically in previous work $[12,13]$. The echo phenomenon can be viewed as a nonlinear mixing of two waves propagating in opposite directions around the ring. If a short duration rf excitation is ap- plied to the beam of the form $\exp \left(i m\left(\omega_{0}+k_{0} \epsilon\right) t\right)$, then the energy dependence of the phase causes the macroscopic perturbation to decay in a Landau damping time. If, however, a second pulse of the form $\exp \left(-i n\left(\omega_{0}+k_{0} \epsilon\right)[t-\delta t]\right)$, is applied after a delay $\delta t$, by virtue of the amplitude nonlinearity, product perturbations of the form $\exp \left(i[m-n]\left(\omega_{0}+k_{0} \epsilon\right) t+\right.$ in $\left.\left(\omega_{0}+k_{0} \epsilon\right) \delta t\right)$, can occur at the difference frequency $(m-$ $n) \omega_{0}$. These second-order perturbations have the property that the energy dependence can disappear at a $t_{E}=n /(n-m) \delta t$, which permits the phase-mixing to be undone, resulting in a reconstruction of a portion of the original perturbation.

A complete analysis of this phenomenon for a coasting beam parallels the parametric coupling anaysis and leads to an explicit expression for the beam current as an integral over the beam distribution function[9].

$$
\begin{aligned}
I_{m}^{(2)}(t)= & i m k_{0} t\left(\frac{e \omega_{0}}{2 \pi}\right)^{3} \sum_{n} \int_{-\infty}^{\infty} d \epsilon \frac{\partial f_{0}}{\partial \epsilon} \\
& \exp \left(i m\left[\omega_{0}+k_{0} \epsilon\right] t-i n\left[\omega_{0}+k_{0} \epsilon\right] \delta t\right. \\
& \left.-\frac{\nu k_{0}^{2} \epsilon_{0}^{2}}{3}\left[m^{2} t^{3}-n^{2} \delta t^{3}\right]\right)
\end{aligned}
$$

where $n$ and $n-m$ are the harmonic numbers of the excitation pulses and $\nu$ is the longitudinal diffusion coef®cient due to scattering or noise. (Wake®eld effects have been ignored to simplify the analysis, but may be readily retained). The characteristic echo response is shown in Fig. 3. The ®rst pulse is at the lower harmonic number $n-m$ while the second pulse occurs at harmonic $n$ after a delay $\delta t$. At time $t_{E}=$ $n /(n-m) \delta t$, an echo is observed whose shape is proportional to $\partial f_{0} / \partial \epsilon$. If a series of various echo delays are chosen, the amplitude envelope maps out a characteristic shape from which the collision frequency $\nu$ can be determined, shown in Fig. 4. Such an experiment was carried out in the Fermilab Antiproton Accumulator yielding a typical momentum diffusion rate of $1 / \tau \approx 3 h r s^{-1}$, which is consistent with the measured heating rate of the momentum cooling system. Thus it is expected that echo decay can be used to measure very weak diffusion rates in beams.

Once veri®ed, bunched beam echoes hold the promise of permitting measurement of a variety of important diffusion mechanisms, such as beam-beam, intra-beam scattering and noise.

\section{Simulations}

Simulations of coherent phenomena in coasting beams have been carried out for a number of years and results relevant to our case were $\circledR$ rst reported in reference 14 . The essential physics is contained in the character of the incremental kicks given to the particle's position and energy per turn, relative to the central momentum particle. These may be expressed in the form

$$
\begin{gathered}
\delta \theta=-\eta(\epsilon) \epsilon \\
\delta \epsilon=\frac{e^{2}}{(2 \pi)^{2} E_{0}} \sum_{n} e^{i n \theta} \int_{-\infty}^{\infty} d \omega Z_{\| n}(\omega) e^{-i \omega t}
\end{gathered}
$$




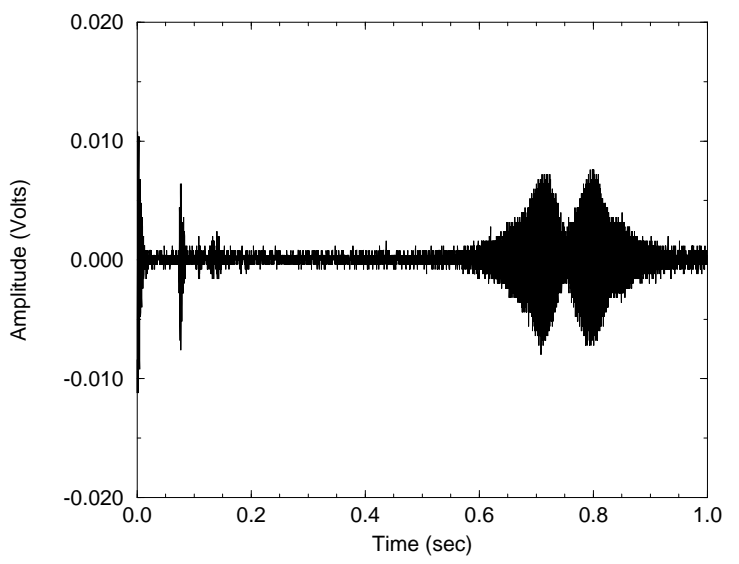

Figure 3: Time evolution of the beam response due to impulse excitation ${ }^{\circledR}$ rst at $n-m=9$ followed by $n=10$ in the Antiproton Accumulator. An echo occurs after a delay $n /(n-m) \delta t$, at the difference harmonic $m=1$.

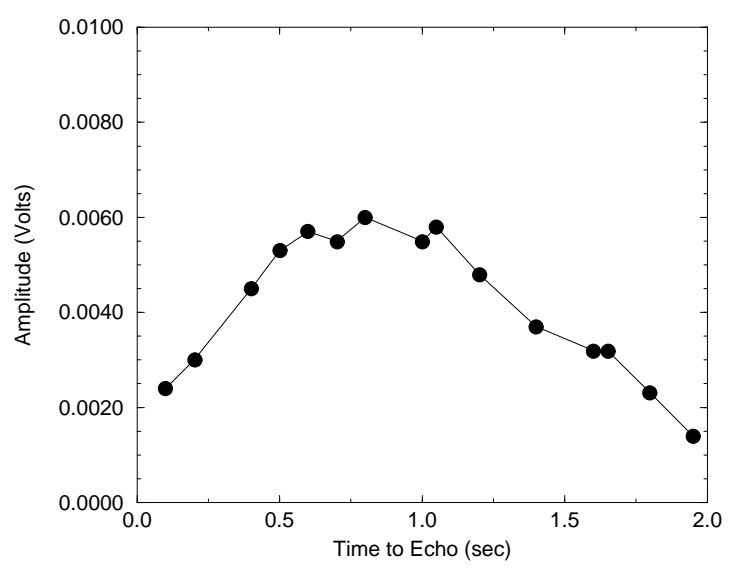

Figure 4: Envelope of the maximum echo amplitude as a function of the applied delay time between excitation pulses. A characteristic decay is observed which depends on weak diffusion processes in the beam.

where $Z_{\|}$is the longitudinal impedance and is the Fourier transform of the wake function given by

$$
Z_{\|}(\omega)=\int_{-\infty}^{\infty} d s e^{\frac{i s}{c}} W_{0}^{\prime}(-s)
$$

In our case, only a ${ }^{\circledR}$ nite collection of modes play a role and we may considerably reduce computation time by restricting the number of modes included in the wake function. The results for a model impedance at $h=10$ demonstrate the tendancy for wave overturning and self-trapping to occur as shown in Fig. 5. A soliton-like structure appears on the low energy side of the distribution function and becomes progressively decelerated in the resulting wake®elds. Such behavior has indeed been observed experimentally in the Main Ring[1]. The macroscopic current

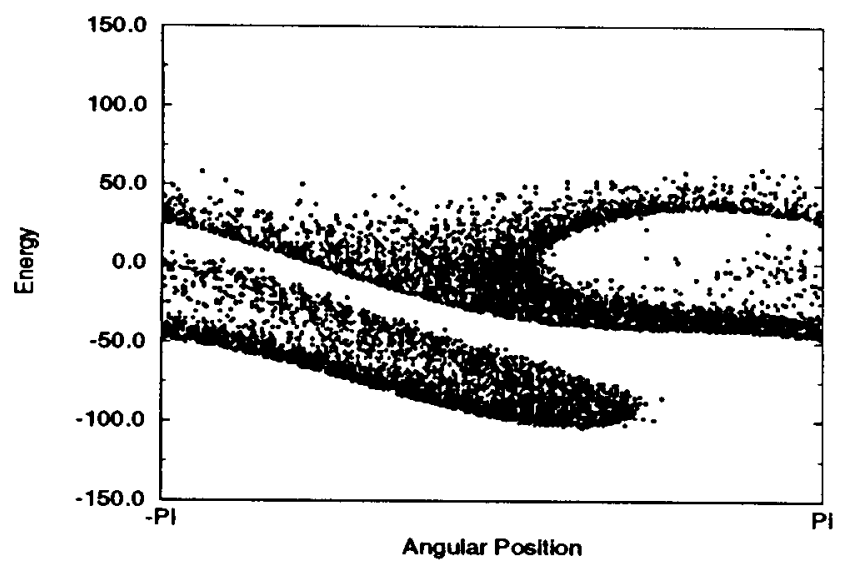

Figure 5: Phase space $(\epsilon-\theta)$ diagram of the particle simulation after initial saturation and wave-overturning has taken place. Soliton-like structures have formed on the low energy side of the distribution.

appears to oscillate in a manner similar to that observed in Fig. 1. Each bunch thus formed undergoes further bifurcation until the momentum spread has increased to the point where the beam has become marginally stable. We can associate these effects to the tendancy to couple power from the initially unstable frequencies over a wide bandwidth of revolution harmonics which then damp via Landau damping, thereby heating the beam. It is evident from the phase-space pictures that the spectral content shows considerable broadening as the instability approaches saturation.

Simulations have also been used to successfully model the parametric coupling process showing the observed sequential coupling to lower sidebands; moreover, the excitation of echoes has been simulated including the effects of wakeßelds and diffusion. Work is underway to permit quantitative determination of the machine impedance from the measurements of nonlinear behavior.

\section{Summary and Conclusions}

We have carried out a series of experiments which have indicated the presence of longitudinal three- wave parametric coupling for beams near marginal stability. The response in the time domain shows a regular causal coupling from high frequency toward lower frequencies, accompanied by waveoverturning and self- trapping of the beam particles.

To explain these effects, we have developed a perturbation model based on the Vlasov equation which explains the singlesided coupling as a manifestation of phase and frequency matching among the coupled modes. In addition, the thresholds for these phenomena are determined by Landau damping rates, which in the case of our observed non-Gaussian distributions, are vanishingly small. In the case of multiple modes, the coupling can proceed as a regular cascade through many harmonics. As such, synchrotrons represent an ideal test bed for studying such coherent phenomena, and the information 
can be used, in principle, as a diagnostic to determine machine impedance and other dynamical variables of the device. Work is in progress to quantify the results presented here.

In addition, the phenomenon of beam echoes can be interpreted as a form of parametric coupling and we have observed classic echo formation in our beams. We have derived an expression which relates the observed echo decay to the rate of diffusion in the beam and anticipate that this will become a viable means of characterizing beam diffusion.

Finally, we have invoked a full-particle simulation to study the strongly nonlinear aspects of the mode excitation and saturation. We have identi®ed the onset of nonlinear Landau damping and the formation of soliton- like structures that has been studied in the context of narrow-band instability. We suggest that a form of resonant mode coupling studied here may play a role in the emittance growth of unbunched as well as bunched beams where the coupling occurs among various synchrtron modes. It is also likely that these phenomena are more widespread that previously thought, due in part to the strongly non-Gaussian character of the momentum distribution in our machines.

\section{Acknowledgements}

The authors would like to acknowledge fruitful discussions with J. Cary, experimental help from G. P. Jackson and S. Assadi and assistance from A. Gerasimov L. Michelotti, J. Holt and $\mathrm{J}$. Beda in carrying out the simulations.

\section{References}

[1] L. K. Spentzouris, P. L. Colestock and F. Ostiguy, this conference

[2] A. Faltens, E. C. Hartwig, D. M\$̀nl and A. M. Sessler, 8th Int. Conf. on High Energy Accel.. CERN p 338, (1971).

[3] E. Keil and W. Schnell, CERN/ISR/TH/RF/ 69-48 (1969)

[4] P. L. Colestock, G. P. Jackson and L. K. Klamp, Proc. 3rd Eur. Particle Accelerator Conf, Berlin, Vol. 1, p. 126 (1992).

[5] R. Z. Sagdeev, A. A. Galeev, T. M. O'Neil and D. L. Book, Nonlinear Plasma Theory, W. A. Benjamin, Inc., New York (1969) and references contained within.

[6] A. S. Bakai, Nuclear Fusion, Vol. 10, p. 53, (1970).

[7] T. M. O'Neil, Phys. Fluids, 8, 12, (1965).

[8] T. M. O'Neil, J. H. Winfrey and J. H. Malmberg, Phys. Fluids, Vol. 14, 6, p. 1204, (1971).

[9] L. Spentzouris, P. L. Colestock and F. Ostiguy, (submitted for publication).

[10] E. L. Hahn, Phys. Rev.,80, 4 (1950).

[11] R. W. Gould, et. al., Phys. Rev. Lett., 19, 5, (1967).
[12] G. V. Stupakov and K. Kauffmann, SSCL-587 (1992).

[13] N. Mahale, et. al., SSCL-N-817 (1993).

[14] E. Keil and E. Messerschmid, Nucl. Inst. and Methods, Vol. 128, p. 203, (1975). 